\title{
'Treasure' in garbage bags: extracellular vesicles based biomarker for neurological diseases
}

\author{
Ashish Kumar, Gagan Deep \\ Department of Cancer Biology, Wake Forest School of Medicine, Atrium Health Wake Forest Baptist, Winston-Salem, NC, USA \\ Correspondence to: Gagan Deep. Department of Cancer Biology, Wake Forest School of Medicine, Atrium Health Wake Forest Baptist, Medical \\ Center Boulevard, Hanes 5048, Winston-Salem, NC 27157, USA. Email: gdeep@wakehealth.edu. \\ Comment on: Saeedi S, Nagy C, Ibrahim P, et al. Neuron-derived extracellular vesicles enriched from plasma show altered size and miRNA cargo as a \\ function of antidepressant drug response. Mol Psychiatry 2021. [Epub ahead of print]. doi: 10.1038/s41380-021-01255-2.
}

Received: 07 November 2021; Accepted: 17 November 2021; Published: 30 December 2021.

doi: $10.21037 /$ exrna-21-25

View this article at: https://dx.doi.org/10.21037/exrna-21-25

\section{Introduction}

Extracellular vesicles (EVs) are micro- to nano-sized membrane-bound vesicles that are secreted by all cell types except viruses as part of their normal physiological function to maintain cellular homeostasis. Initially, EVs were considered as 'garbage bags' that cells utilize to throw their metabolic waste out. However, these vesicles were later recognized to play a crucial role in intercellular communication by transferring various biomolecules (loaded as cargos) such as proteins, nucleic acids (mostly non-coding RNAs), lipids and metabolites. Majorly EVs are divided into three subclasses: apoptotic bodies, microvesicles (MVs), and exosomes. Apoptotic bodies are released from the cells undergoing programmed cell death and range from $\sim 50$ to $5 \mu \mathrm{m}$ in size $(1,2)$. MVs are $\sim 100$ to $\geq 1,000 \mathrm{~nm}$ in size and arise by outward budding of the plasma membrane (3). Exosomes are derived from the endosomal system and released to the extracellular environment as $\sim 30-150 \mathrm{~nm}$ size vesicles. EVs isolated from in vivo system cannot be distinguished if they are generated from the endosomal pathway or released from the outward budding of the plasma membrane. Also, due to their overlapping size and lack of specific biomarkers, it is difficult to discriminate these vesicles and, therefore, collectively referred as EVs. In the last decade, small EVs (sEVs), mainly enriched in exosomes, have garnered much attention for their role as biomarkers for several diseases, including cancer $(4,5)$, cardiovascular disease $(6,7)$, diabetes, and obesity $(8,9)$, inflammation (10) and psychiatric disorders $(11,12)$. The possible isolation of sEVs from almost every biological fluid and the recent methodological advancement to isolate cell type-specific sEVs pose them as an attractive target for the development of non/less invasive biomarkers for complicated diseases like psychiatric disorders. Analyzing the cargos of neuron-derived EVs (NDEVs) have been shown to predict the development of Alzheimer's disease (AD) up to 10 years before the clinical onset (13). Ledreux et al. reported that the NDEVs isolated from the individuals with Down syndrome and AD are loaded with p-Tau and have the tau seeding capacity to spread tau pathology in the mouse brain (14). Recently, our group also reported the utility of NDEVs and other brain cell-derived EVs in identifying the neurodegenerative and pro-inflammatory effects of oxycodone self-administration in cynomolgus monkeys (15).

\section{Does EV size matter?}

A recent elegantly designed study by Saeedi $e t$ al. showed the potential of NDEVs in identifying the effect of an antidepressant drug in major depressive disorder (MDD) patients (16). Authors in that study isolated $\mathrm{EV}$ s from the plasma of control and MDD subjects and enriched for NDEVs using neural cell adhesion molecule L1CAM, both before (T0) and after 8 weeks of therapy (T8) with antidepressant drug escitalopram. The characterization of EVs and NDEVs presented some interesting observations, such as; the NDEVs were smaller in size than total EVs and the size of the NDEVs changed as a function of treatment response measured by change ( $>50 \%$ decrease) in 
Montgomery-Asberg Depression Rating Scale (MADRS) score. Interestingly, patients responding positively to the therapy showed a significant increase in NDEVs in the size range of 101 to $150 \mathrm{~nm}$, while the concentration of smaller size NDEVs decreased. The authors also confirmed the observation in two separate cell lines (human neural progenitor Rencells and HEK293T cells) as treatment of these cells with the same antidepressant drug increased the size and amount of the EVs. This study proposes that smaller EVs might be loaded with less cargo suggesting a reduction of communication via NDEVs cargo in untreated MDD patients, which is rescued upon escitalopram response.

In the EVs research field, the isolation methodology and EVs characterization are of foremost importance. Use of more than one method for EVs characterization may be opted when drawing any conclusion based on the change in their size and concentration. In this study (16), two methods for EVs characterization were employed, namely, tunable resistive pulse sensing (TRPS) (Izon Science) and nCS1 instrument (Spectradyne). However, both the instruments work on the same resistive pulse sensing technology. It would have been interesting to observe if the reported changes in the size of NDEVs are consistent using a different approach i.e., analyzing light scattering and Brownian motion with nanoparticle tracking analysis or other methods suggested by van der Pol et al. (17). Although authors judiciously controlled the TRPS measurements recorded at two different time points by adjusting the measurements at $\mathrm{T} 0$ with the mean and standard deviation of the controls at $\mathrm{T} 8$.

Interestingly, most of the studies conducted on sEVs to represent them as potential biomarkers for diverse diseases are focused on their cargos. Some of the studies also reported that following chemical or physiological stress amount of EVs secretion increases $(18,19)$. Though most of these studies were performed in vitro and any conclusion drawn on the basis of in vitro condition may not extrapolate in the in vivo system. However, there are not many studies that suggest a significant change in sEVs size following drug treatment and relate that as a function of treatment response. This study by Saeedi et al. (16) presented an increase in NDEVs size as a response to antidepressant drug treatment for 8 weeks in the MDD cohort and then confirmed that observation in two cell lines in cell culture. This observation might be reasonable for a drug that can target proteins involved in EV biogenesis. However, given the fact that escitalopram belongs to the selective serotonin reuptake inhibitors (SSRIs) group of drugs and is not reported to affect the EV biogenesis, the results from this study need to be further pursued to identify the underlying mechanisms. It is also interesting to note that the SSRIs affect the neurons expressing the serotonin transporter, and authors suggested this as a possible hypothesis to explain NDEVs size change; however, the size change in the NDEVs was reported in L1CAM positive NDEVs, which is a general marker for the neurons and not specific to serotonergic neurons or brain neurons.,

The enrichment of cell type-specific EVs from plasma has provided an immense possibility to predict the pathophysiological state of the parent cell, especially in deeper tissues or for hard-to-access organs. However, one should be cautious while selecting the marker protein to isolate a specific EV subpopulation. Using two or more surface markers could be a better approach to confirm the changes in cell- or tissue-specific EVs. The selected markers could either be used sequentially to isolate cell type-specific EVs, or the effect observed with the selected first marker used for EVs isolation could be verified using a second surface marker. In the study by Saeedi et al. (16), NDEVs were enriched using neuronal marker L1CAM, and then a few of the key observations were further verified using another neuronal marker synaptosome-associated protein 25 (SNAP25) in an independent cohort. This is certainly convincing considering a recent conflicting report on L1CAM presence on EVs in plasma or CSF (20), though it is widely reported to enrich NDEVs. Compared to L1CAM, SNAP25 expression is mainly limited to brain tissues, and it will be interesting to see the relative abundance of SNAP25+ EVs in plasma and their utility in characterizing biomarkers for neurological and psychiatric diseases.

\section{MicroRNAs (miRNAs) cargos in EV}

Analyzing the cargos of EVs seems more reasonable as those could possibly reflect the pathophysiological state of the parent cells. Many studies have shown that analyzing the EV cargos (mainly proteins and miRNAs) can predict the onset, progression, and treatment response of psychological disorders $(13,15,21)$. The pathological proteins associated with neurodegenerative diseases like $\alpha$-synuclein, $\mathrm{p}$-tau/ tau, and amyloid $\beta$ could be transported through EVs to nonpathological areas promoting the progression of disease $(22,23)$. miRNAs loaded in EVs are relatively more stable compared to free circulatory miRNAs and are also involved 
in the pathogenesis of neurodegenerative and psychiatric disorders $(12,24)$. Characterizing the miRNAs loaded in EVs could serve as a signature to represent the host cell's status. Though it is also important to consider that the circulatory miRNAs are not always packed in EVs, they can freely circulate in the biofluids. While analyzing the miRNAs in EVs is important and informative, great care should be taken to isolate EVs from the biofluids as it is important to remove miRNAs that might be arbitrarily sticking to the EVs membrane. In the study by Saeedi et al. (16), the isolated NDEVs were treated with RNase A so that the analyzed miRNAs will represent the tissue of origin and not some random miRNAs non-specifically sticking to the EVs surface. The specificity of the analyzed miRNA expression was further confirmed by comparing them with the miRNAs isolated from the brain tissuederived EVs. The study showed that the analyzed miRNAs in the NDEVs of MDD patients actually mirror the EV miRNAs extracted from brain tissue with $89 \%$ overlap in the number of identified miRNAs. Interestingly, these miRNAs identified in NDEVs were shown to represent the tissue identity as they did not overlap at all with the miRNAs reported from the saliva EVs (25).

Furthermore, analyzing the miRNAs cargos of the NDEVs isolated from controls and MDD patients, both before and after 8 weeks of therapy, authors identified 75 miRNAs using small RNA sequencing analysis that showed at least three counts in $70 \%$ of a given group (control, responders and non-responders). The authors compared if the expression of these miRNAs altered with the treatment response. The analysis identified 9 miRNAs (miR-423-3p, miR-191-5p, miR-486-5p, miR-30d-5p, miR-425-5p, miR25-3p, miR-21-5p, miR-335-5p, and miR-126-5p) changed from before to after therapy with statistical significance based on small RNA sequencing data. The backward stepwise regression analysis was performed to determine if any combination of these miRNAs can predict the response of the therapy ('responders' vs. 'non-responders'). The authors identified the combination of three miRNAs miR-21-5p, miR-30d-5p, and miR-486-5p changed over therapy and can predict the treatment response. However, the direction of change of these miRNAs was not reported (increase or decrease with therapy). Also, the change in expression of these three miRNAs was validated using qPCR, and expression of only miR-21-5p showed a significant correlation with the sequencing data. One important thing to note while using qPCR to validate the sequencing data is the selection of normalizing control.
The authors in this study used miR-103a-3p and miR-9-5p as normalizing control to calculate the relative fold change $\left(2^{-\Delta \Delta C \mathrm{C}}\right)$. The selection of miR-103a-3p seemed reasonable as it was based on its stability as assessed using NormFinder. Although miR-9-5p displays a conserved expression pattern in the central nervous system, its expression in the brain is reported to be altered with stress, and it is involved in several neurodegenerative diseases (26). The selection of stable miRNA that can be used as normalizing control for the qPCR data is very context-dependent as miRNAs expression, especially in the EVs, can change in different disease conditions. It will certainly not be equitable to select one miRNA as a universal normalizing control even if it shows conserved expression in a particular tissue. The selection of miRNA should be based on its stability in that particular cohort in a given disease context. Alternatively, if it is difficult to identify the stable miRNA for normalization of the data, external miRNA control (spike-in control) can be used. Caenorhabditis elegans miRNA-39-3p (cel-miR39-3p) can be used for this purpose and can be mixed with EVs before the isolation of RNA. Though it mainly serves as a control for the efficiency of RNA isolation or cDNA synthesis, in certain conditions it can also be used as a normalizing control.

\section{Validation is important}

It is also important to cogitate if the observed change in the expression of miRNAs is consistent and does not change with the choice of marker used to isolate NDEVs. To confirm the expression of miRNAs observed in L1CAM positive EVs, authors in this study used a second marker SNAP25 to isolate NDEVs from a separate validation cohort and showed the consistency in the expression of two miRNAs (miR-486-5p and miR-30-5p), out of three miRNAs (miR-21-5p showed no change) used as a combination to predict 'responders' vs. 'non-responders'. However, the ratio of change on these two miRNAs at T8/ $\mathrm{T} 0$ in responders is close to one, which suggests that their expression did not change over time in the patients who responded to therapy, though it decreases (less than one) in non-responders. Since the change in miRNA expression in NDEVs represents the brain status, it is important to relate their functional consequences and involvement in treatment response (or disease progression). Enrichment of target prediction of these three miRNAs showed that their targets are enriched in the brain tissue. This corroborates the authors' other findings in this study that the miRNAs 
identified in NDEVs significantly overlap with the miRNAs from brain tissue-derived EVs.

\section{Clinical significance}

The analysis of cargos of EVs (or cell type-specific EVs) is certainly exciting; however, whether those could be of any clinical significance or provide any translational application is also critically important. If the cargos of EVs can distinguish between 'control' or 'diseased' subjects or treatment 'responders' vs. 'non-responders', it will be of high translational significance. The comparable changes in the expression of targets of identified miRNAs in this study with the brain tissue (anterior cingulate cortex) of individuals who died by suicide during an episode of major depression represent the high clinical prominence of NDEV miRNAs. Interestingly, the mRNA sequencing of the brain tissue of control and those who died by suicide show differential expression, which overlaps with the identified mRNA targets of miRNAs loaded in NDEVs. Though the data presented in this study compared the subjects before and after the therapy, it will be very interesting to see if it is possible to identify the patients who may respond to therapy by analyzing the NDEVs' cargo (or possibly size) at the start of the therapy.

\section{Conclusions}

In conclusion, Saeedi and colleagues (16) have presented an exciting study to show the utility of NDEVs to identify the response to antidepressant therapy. They also presented an interesting observation that the size of the NDEVs is smaller compared to total EVs, and NDEVs size is modulated as a response to therapy. The use of more than one marker to isolate NDEVs (L1CAM and SNPA25) in this study is also a step in the right direction. It will be interesting to see whether NDEVs isolated using these two markers are overlapping or distinct. Most importantly, miRNA analysis of NDEVs and their targets show a similar change in the brain tissue of the patients who died by suicide during MDD. This suggests that analysis of miRNA cargos in plasma NDEVs could serve as a proxy marker to predict the pathophysiological status of the brain cells and treatment response. These findings and approaches are certainly applicable to other neurological and psychiatric disorders.

\section{Acknowledgments}

Funding: None.

\section{Footnote}

Provenance and Peer Review: This article was commissioned by the editorial office, ExRNA. The article has undergone external peer review.

Conflicts of Interest: Both authors have completed the ICMJE uniform disclosure form (available at https://dx.doi. org/10.21037/exrna-21-25). The authors have no conflicts of interest to declare.

Ethical Statement: The authors are accountable for all aspects of the work in ensuring that questions related to the accuracy or integrity of any part of the work are appropriately investigated and resolved.

Open Access Statement: This is an Open Access article distributed in accordance with the Creative Commons Attribution-NonCommercial-NoDerivs 4.0 International License (CC BY-NC-ND 4.0), which permits the noncommercial replication and distribution of the article with the strict proviso that no changes or edits are made and the original work is properly cited (including links to both the formal publication through the relevant DOI and the license). See: https://creativecommons.org/licenses/by-nc-nd/4.0/.

\section{References}

1. György B, Szabó TG, Pásztói M, et al. Membrane vesicles, current state-of-the-art: emerging role of extracellular vesicles. Cell Mol Life Sci 2011;68:2667-88.

2. Battistelli M, Falcieri E. Apoptotic Bodies: Particular Extracellular Vesicles Involved in Intercellular Communication. Biology (Basel) 2020;9:21.

3. Chen Y, Li G, Liu ML. Microvesicles as Emerging Biomarkers and Therapeutic Targets in Cardiometabolic Diseases. Genomics Proteomics Bioinformatics 2018;16:50-62.

4. Panigrahi GK, Deep G. Exosomes-based biomarker discovery for diagnosis and prognosis of prostate cancer. Front Biosci (Landmark Ed) 2017;22:1682-96.

5. Zhang L, Yu D. Exosomes in cancer development, metastasis, and immunity. Biochim Biophys Acta Rev 
Cancer 2019;1871:455-68.

6. Zhang Y, Hu YW, Zheng L, et al. Characteristics and Roles of Exosomes in Cardiovascular Disease. DNA Cell Biol 2017;36:202-11.

7. Sahoo S, Losordo DW. Exosomes and cardiac repair after myocardial infarction. Circ Res 2014;114:333-44.

8. Castaño C, Novials A, Párrizas M. Exosomes and diabetes. Diabetes Metab Res Rev 2019;35:e3107.

9. Pardo F, Villalobos-Labra R, Sobrevia B, et al. Extracellular vesicles in obesity and diabetes mellitus. Mol Aspects Med 2018;60:81-91.

10. Console L, Scalise M, Indiveri C. Exosomes in inflammation and role as biomarkers. Clin Chim Acta 2019;488:165-71.

11. Saeedi S, Israel S, Nagy C, et al. The emerging role of exosomes in mental disorders. Transl Psychiatry 2019;9:122.

12. Aharon A, Spector P, Ahmad RS, et al. Extracellular Vesicles of Alzheimer's Disease Patients as a Biomarker for Disease Progression. Mol Neurobiol 2020;57:4156-69.

13. Fiandaca MS, Kapogiannis D, Mapstone M, et al. Identification of preclinical Alzheimer's disease by a profile of pathogenic proteins in neurally derived blood exosomes: A case-control study. Alzheimers Dement 2015;11:600-7.e1.

14. Ledreux A, Thomas S, Hamlett ED, et al. Small NeuronDerived Extracellular Vesicles from Individuals with Down Syndrome Propagate Tau Pathology in the Wildtype Mouse Brain. J Clin Med 2021;10:3931.

15. Kumar A, Kim S, Su Y, et al. Brain cell-derived exosomes in plasma serve as neurodegeneration biomarkers in male cynomolgus monkeys self-administrating oxycodone. EBioMedicine 2021;63:103192.

16. Saeedi S, Nagy C, Ibrahim P, et al. Neuron-derived extracellular vesicles enriched from plasma show altered size and miRNA cargo as a function of antidepressant drug response. Mol Psychiatry 2021. doi: 10.1038/s41380-021-

doi: 10.21037/exrna-21-25

Cite this article as: Kumar A, Deep G. 'Treasure' in garbage bags: extracellular vesicles based biomarker for neurological diseases. ExRNA 2021;3:14.
01255-2.

17. van der Pol E, Coumans FA, Grootemaat AE, et al. Particle size distribution of exosomes and microvesicles determined by transmission electron microscopy, flow cytometry, nanoparticle tracking analysis, and resistive pulse sensing. J Thromb Haemost 2014;12:1182-92.

18. Kumar A, Deep G. Exosomes in hypoxia-induced remodeling of the tumor microenvironment. Cancer Lett 2020;488:1-8.

19. Cesselli D, Parisse P, Aleksova A, et al. Extracellular Vesicles: How Drug and Pathology Interfere With Their Biogenesis and Function. Front Physiol 2018;9:1394.

20. Norman M, Ter-Ovanesyan D, Trieu W, et al. L1CAM is not associated with extracellular vesicles in human cerebrospinal fluid or plasma. Nat Methods 2021;18:631-4.

21. Lim CZJ, Zhang Y, Chen Y, et al. Subtyping of circulating exosome-bound amyloid $\beta$ reflects brain plaque deposition. Nat Commun 2019;10:1144.

22. D'Anca M, Fenoglio C, Serpente M, et al. Exosome Determinants of Physiological Aging and Age-Related Neurodegenerative Diseases. Front Aging Neurosci. 2019;11:232.

23. Jiang R, Rong C, Ke R, et al. Differential proteomic analysis of serum exosomes reveals alterations in progression of Parkinson disease. Medicine (Baltimore). 2019;98(41):e17478.

24. Ge Q, Zhou Y, Lu J, et al. miRNA in plasma exosome is stable under different storage conditions. Molecules 2014;19:1568-75.

25. Ogawa Y, Taketomi Y, Murakami M, et al. Small RNA transcriptomes of two types of exosomes in human whole saliva determined by next generation sequencing. Biol Pharm Bull 2013;36:66-75.

26. Coolen M, Katz S, Bally-Cuif L. miR-9: a versatile regulator of neurogenesis. Front Cell Neurosci. 2013;7:220. 